\title{
THz Switching of Antiferromagnets and Ferrimagnets
}

\author{
S. Wienholdt, D. Hinzke, and U. Nowak \\ Fachbereich Physik, Universität Konstanz, Konstanz D-78457, Germany \\ (Received 19 December 2011; revised manuscript received 9 March 2012; published 13 June 2012)

\begin{abstract}
Using analytical calculations as well as computer simulations, we show that antiferromagnets can be switched on a time scale of picoseconds using $\mathrm{THz}$ laser pulses only. This all-optically triggered switching mechanism rests on the coordinated dynamics of the two interacting sublattices with an inertial character. We calculate the resonance frequencies in the nonlinear regime, the orbits, and estimate the field strength required for switching analytically. Furthermore, we demonstrate that ferrimagnets can be switched similarly at their compensation point.
\end{abstract}

DOI: 10.1103/PhysRevLett.108.247207

PACS numbers: 75.78.Jp, 75.60.Jk, 75.10.Hk

One of the most interesting problems in modern magnetism is the question on what time scales and by what means magnetization switching can be achieved. The first observation of subpicosecond demagnetization of a thin $\mathrm{Ni}$ film excited by a $60 \mathrm{fs}$ laser pulse [1] suggested already that such a pulse is a powerful stimulus for ultrafast magnetization dynamics. Later on it was even demonstrated that a 40 fs circularly polarized pulse can reverse the magnetization in a ferrimagnetic film [2]. Recently, it was argued [3] that this all-optical switching of the magnetization proceeds via a novel linear reversal mechanism [4] where the heat pulse from the laser first brings the medium into a highly nonequilibrium state with no net magnetization, while the circularly polarized pulse acts as an effective magnetic field via the inverse Faraday effect controlling the linear reversal (for a review on all-optical manipulation of magnetic order see Ref. [5]).

All-optical magnetization switching was so far only demonstrated in ferrimagnets, so that currently many investigations focus on the understanding of the special behavior of antiferromagnets and ferrimagnets, the dynamics of both of which is governed by the two interacting sublattices. Due to the relatively strong exchange interaction, the dynamics of an antiferromagnetic exchange mode is much faster than ferromagnetic dynamics [6,7]. Furthermore, in antiferromagnets, the analogon to an inertial behavior does exist with possible switching mechanisms, which can be triggered by short field pulses though the switching itself takes much longer [8]. The new development of ultrashort, single-cycle laser pulses in the $\mathrm{THz}$ regime $[9,10]$ opens up a further, new path for the excitation of magnetic materials. Recently, Kampfrath et al. [11] demonstrated the excitation of spin waves in the antiferromagnet $\mathrm{NiO}$ with a single-cycle $\mathrm{THz}$ laser pulse, where the excitation was solely driven by the $B$-field component of the electromagnetic wave.

In this Letter, we show that $\mathrm{THz}$ laser pulses can in principle be used to even switch the sublattice magnetization of antiferromagnets on a picosecond time scale. This switching mechanism rests on the coordinated, nonlinear dynamics of the two interacting sublattices, with an inertial component. We calculate the resonance frequencies in the nonlinear regime, the orbits, and the field strength required for switching analytically. Furthermore, using computer simulations we show that ferrimagnets at the compensation point can be switched similarly.

We investigate the dynamics of antiferromagnets and ferrimagnets within the framework of a classical, atomistic spin model. The Hamiltonian for unit vectors, $\mathbf{S}_{i}$, representing the normalized magnetic moment of the $i$ th atom with magnetic moment $\mu_{s}^{i}$ reads [12]

$$
\mathcal{H}=\sum_{\langle i<j\rangle} J_{i j} \mathbf{S}_{i} \cdot \mathbf{S}_{j}-\sum_{i} d_{z}\left(S_{i}^{z}\right)^{2}-\mu_{s}^{i} \mathbf{B} \cdot \sum_{i} \mathbf{S}_{i}
$$

The first term describes the Heisenberg exchange with exchange constants $J_{i j}$ taking into account the nearest neighbors (NN) and the next nearest neighbors (NNN). The second term represents a uniaxial anisotropy with anisotropy constant $d_{z}$. The last term is the Zeeman energy with a time-dependent external magnetic field $\mathbf{B}(t)$.

As an equation of motion, we consider Langevin dynamics, i.e., the stochastic Landau-Lifshitz-Gilbert equation. Thermal fluctuations are included via an additional white-noise term. The coupling of the system to the heat bath is described by a phenomenological, microscopic damping constant $\alpha$ [12].

We start our investigations with simulations of the dynamics of antiferromagnets at $0 \mathrm{~K}$ considering a simple toy model with 64 spins arranged on a cubic lattice with periodic boundary conditions. Each spin has magnetic moment $\mu_{s}$ and gyromagnetic ratio $\gamma$. We introduce an antiferromagnetic coupling for $\mathrm{NN}\left(J^{\mathrm{NN}}>0\right)$ and a ferromagnetic coupling for $\mathrm{NNN}\left(J^{\mathrm{NNN}}=-0.1 J^{\mathrm{NN}}\right)$. The anisotropy constant is chosen as $d_{z}=0.01 J^{\mathrm{NN}}$, and $\alpha$ as 0.001 . For simplicity, we excite the antiferromagnet with rectangular field pulses $B_{x}(t)$ in $x$ direction, perpendicular to the easy axis (Fig. 1). The pulse duration is around $t \approx \pi / \omega^{\mathrm{AFMR}}$, where $\omega^{\mathrm{AFMR}}$ is the antiferromagnetic resonance (AFMR) frequency. These pulses trigger a precessive motion of the magnetization of the two sublattices, $\mathbf{M}_{1}$ and $\mathbf{M}_{2}$. The effective fields of the two 
a)

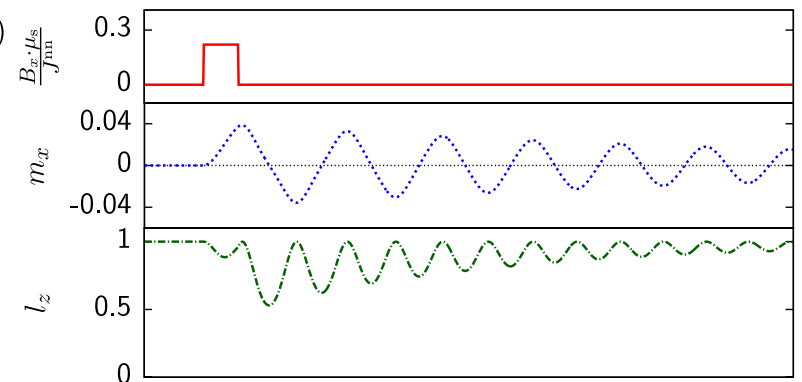

b)

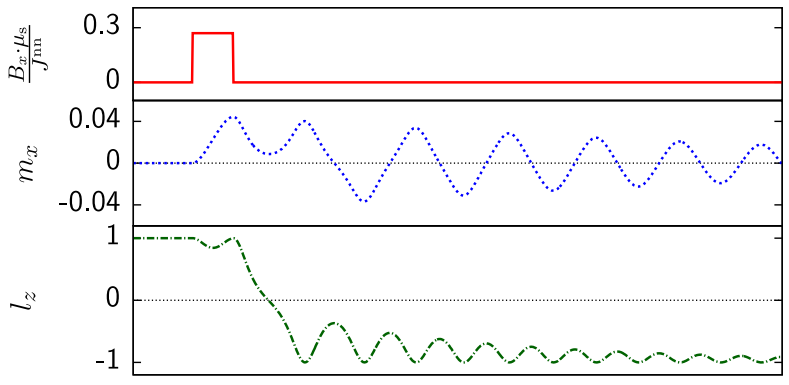

c)

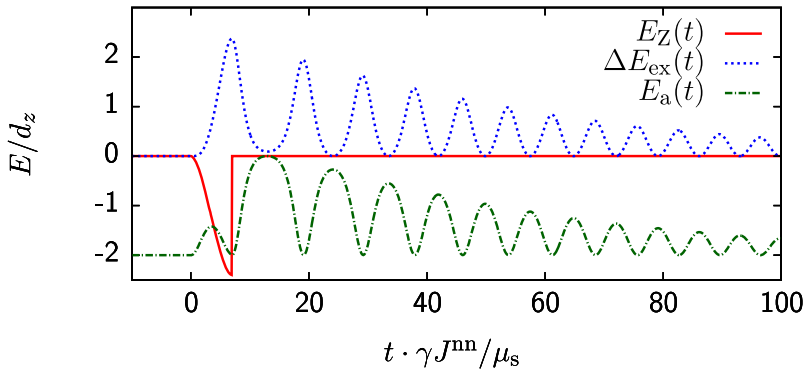

FIG. 1 (color online). Excitation of an antiferromagnet with a ps magnetic field pulse. (a) $l_{z}$ shows inertial behavior. (b) Excitation triggers inertial switching. (c) Energies during switching process.

sublattices have opposite signs, and so the subblatice magnetizations precess in the opposite direction. Therefore, the total normalized magnetization, $\mathbf{m}=\left(\mathbf{M}_{1}+\mathbf{M}_{2}\right) /\left(2 M_{0}\right)$ with $M_{0}=\left|\mathbf{M}_{1}\right|=\left|\mathbf{M}_{2}\right|$, increases in field direction [Fig. 1(a)], while $m_{y}$ and $m_{z}$ remain zero.

While $m_{x}$ shows a damped oscillation after the field has been turned off, the antiferromagnetic order parameter $\mathbf{l}=$ $\left(\mathbf{M}_{1}-\mathbf{M}_{2}\right) /\left(2 M_{0}\right)$ shows a surprising behavior [Fig. 1(a)]: there is only a small disturbance of the antiferromagnetic order as long as the field is still on but the disturbance increases after the field has been turned off. One can exploit this behavior to trigger a so-called "inertial" switching [8]; i.e., one can excite the system with a short field pulse and the system will switch on its own after the field is already gone [see Fig. 1(b), and Ref. [13] for a short movie]. The switching can also be observed in the total magnetization where it leads to a phase shift [Fig. 1(b)].

The inertial behavior can be explained through the interplay of the different energy contributions during switching [Fig. 1(c)]. As long as the field is turned on, the Zeeman energy, $E_{\mathrm{Z}}$, decreases and is converted into increasing exchange, $E_{\mathrm{ex}}$, and anisotropy energy, $E_{a}$. Afterwards, in zero field, the energy oscillates between anisotropy and exchange as in a mechanical oscillator where the energy oscillates between potential and kinetic energy. The analogon of the kinetic energy here is the exchange energy between the two sublattices. Switching occurs if there is sufficient energy stored in the exchange to overcome the anisotropy barrier. In case the damping is high enough so that the system cannot pass the energy barrier a second time, it will relax into its new equilibrium state with switched sublattices [Fig. 1(b)]. For higher excitation or lower damping, the system can also switch several times until it finally relaxes into one of the equilibrium states.

In order to get a deeper understanding of the inertial switching, the Landau-Lifshitz equation of motion is solved analytically for two normalized macro spins, $\mathbf{m}_{1}$, $\mathbf{m}_{2}$, representing the two sublattices with effective exchange constant $\tilde{J}$ neglecting damping and thermal fluctuations [13]. We assume that the dynamics starts from the antiferromagnetic ground state and a magnetic field $\mathbf{B}=$ $B \mathbf{e}_{x}$ is applied perpendicularly to the easy $z$ axis. For symmetry reasons, the $y$ and $z$ components of $\mathbf{m}$ and the $x$ component of $\mathbf{I}$ remain zero. Hence, one can use the coordinates $\mathbf{m}_{1}=(\mathbf{m}+\mathbf{l})=\left(m_{x}, l_{y}, l_{z}\right)$ and $\mathbf{m}_{2}=$ $(\mathbf{m}-\mathbf{l})=\left(m_{x},-l_{y},-l_{z}\right)$ to derive a system of three coupled differential equations as follows:

$$
\begin{gathered}
\dot{m}_{x}=-2 \gamma d_{z} l_{y} l_{z} / \mu_{s}, \\
i_{y}=\gamma\left(2\left(d_{z}+\tilde{J}\right) m_{x} l_{z}-\mu_{s} B l_{z}\right) / \mu_{s}, \\
i_{z}=-\gamma\left(2 \tilde{J} m_{x} l_{y}-\mu_{s} B l_{y}\right) / \mu_{s} .
\end{gathered}
$$

Analyzing first the dynamics after the field pulse is turned off we define the energy $E$ per spin pair of the initial excitation by the energy difference to the ground state $E:=$ $\tilde{J} \mathbf{m}_{1} \cdot \mathbf{m}_{2}-d_{z}\left(m_{1}^{z}\right)^{2}-d_{z}\left(m_{2}^{z}\right)^{2}+\tilde{J}+2 d_{z}$. Combining Eqs. (2)-(4) and using the chain rule, one finds quadratic equations for combinations of the components of the form $x^{2} / a^{2} \pm y^{2} / b^{2}=1$. It is sufficient to consider the two equations with a plus sign, which describe ellipses, and hence can be parameterized by trigonometric functions using angular variables $\varphi$ and $\theta$, where $\varphi$ is the angle between the projection of $\mathbf{m}_{1}$ to the $x-y$ plane and the $x$ axis, and $\theta$ is the angle between the projection of $\mathbf{m}_{\mathbf{1}}$ to the $y-z$ plane and the $z$ axis as follows:

$$
\begin{gathered}
m_{x}=a_{1} \cos (\varphi(t))=a_{1} \sqrt{1-\left(b_{2} / b_{1}\right)^{2} \sin ^{2}(\theta(t)),} \\
l_{y}=b_{1} \sin (\varphi(t))=b_{2} \sin (\theta(t)), \\
l_{z}=a_{2} \cos (\theta(t))=a_{2} \sqrt{1-\left(b_{1} / b_{2}\right)^{2} \sin ^{2}(\varphi(t))},
\end{gathered}
$$

where $a_{1}=\sqrt{E /\left(2\left(d_{z}+\tilde{J}\right)\right)}$ and $b_{1}=\sqrt{E / 2 d_{z}}$ are the semiaxes of the elliptic equation for $m_{x}$ and $l_{y}$, and $a_{2}=$ $\sqrt{\left(d_{z}+\tilde{J}-E / 2\right) /\left(d_{z}+\tilde{J}\right)}$ and $b_{2}=\sqrt{\left(d_{z}+\tilde{J}-E / 2\right) / \tilde{J}}$ 
are the semiaxes of the elliptic equation for $l_{y}$ and $l_{z}$. Using Eq. (6), one can express $m_{x}$ as a function of $\theta$, and $l_{z}$ as a function of $\varphi$.

To solve Eqs. (2)-(4), we insert Eqs. (5)-(7) into Eq. (2) and get two nonlinear differential equations, which are solved by the Jacobi amplitude function $\operatorname{am}\left(x \mid k^{2}\right)$,

$$
\begin{aligned}
& \varphi(t)=\operatorname{am}\left(2 \frac{\gamma}{\mu_{s}} d_{z} \frac{a_{2} b_{1}}{a_{1}} t \mid\left(b_{1} / b_{2}\right)^{2}\right), \\
& \theta(t)=\operatorname{am}\left(2 \frac{\gamma}{\mu_{s}} \tilde{J} \frac{a_{1} b_{2}}{a_{2}} t \mid\left(b_{2} / b_{1}\right)^{2}\right) .
\end{aligned}
$$

These solutions describe two different modes. For $E<2 d_{z}$, the spins precess around the easy axis [Fig. 2(c)], while for $E>2 d_{z}$, the spins precess around the $x$ axis [Fig. 2(b)].

The Jacobi amplitude function has the property $\operatorname{am}\left(x \mid k^{2}\right)=n \pi$ for $k<1$ and $x=2 n K(k)$. Here, $n \in \mathbb{N}$ and $K(k)$ is the complete elliptic integral of the first kind. With this we can calculate the eigenfrequencies of the system exactly without any linearization. For $E<2 d_{z}$, the spins precess like in Fig. 2(c) with frequency

$$
\omega_{1}^{\mathrm{AFMR}}=\frac{\pi \gamma}{\mu_{s}} \sqrt{d_{z}\left(d_{z}+\tilde{J}\right)-d_{z} E / 2} \frac{1}{K\left(b_{1} / b_{2}\right)},
$$

and for $E>2 d_{z}$, like in Fig. 2(b) with frequency

$$
\omega_{2}^{\mathrm{AFMR}}=\frac{\pi \gamma}{\mu_{s}} \sqrt{\tilde{J} E / 2} \frac{1}{K\left(b_{2} / b_{1}\right)} .
$$

Note that for small energies, Eq. (10) converges to the usual, linearized solution for the AFMR frequency [6].

To solve the differential equation for the excitation process $\left(B_{x} \neq 0\right)$, one has to follow the same procedure. Combining Eqs. (2) and (3) leads to a quadratic equation

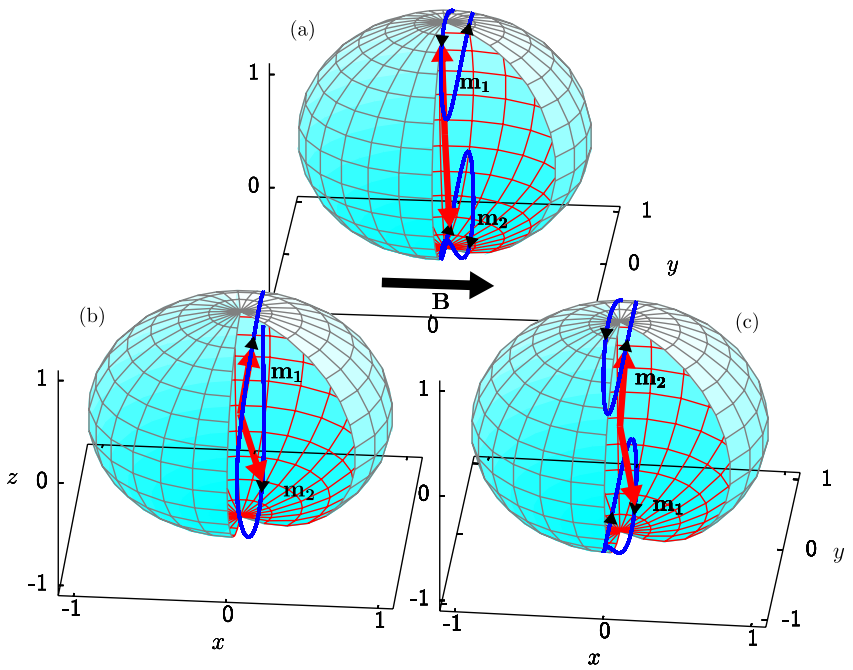

FIG. 2 (color online). (a) Precession of the antiferromagnet during the excitation with a magnetic field $\mathbf{B}$. The lines represent orbits of sublattice magnetizations. (b) Precession in zero field for $E>2 d_{z}$. (c) $E>2 d_{z}$. for $l_{y}$ and $m_{x}$. The difference to the elliptic equation before is that the ellipse is shifted along the field, with its center at $\left(\frac{\mu_{s} B}{2\left(d_{z}+\tilde{J}\right)}, 0\right)$ and its semiaxis in $x$ direction as $\frac{\mu_{s} B}{2\left(d_{z}+\tilde{J}\right)}$ [Fig. 2(a)].

We find a minimum of Zeeman energy for a maximal magnetization in field direction, which is at $m_{x}=\frac{\mu_{s} B}{d_{z}+\tilde{J}}$. As this is the energy stored in the system after the field is turned off, we can calculate a lower limit of the field for reaching the energy needed for switching $\left(E=2 d_{z}\right)$ as follows:

$$
\mu_{s} B_{\min }=\sqrt{d_{z}\left(d_{z}+\tilde{J}\right)} .
$$

The minimum of the Zeeman energy is reached after $1 / 2$ precession. To get the optimal duration time for a pulse, we calculate the frequencies of the precession with perpendicularly applied field [Fig. 2(a)]. Following the same procedure as before, we get a nonlinear differential equation for $\varphi(t)$, which now depends on the external field. In a good approximation [13], the Jacobi amplitude function also solves this equation and we get for the frequencies,

$$
\omega^{B}=\frac{\pi \gamma}{\mu_{s}} \sqrt{d_{z}\left(d_{z}+\tilde{J}\right)-\frac{d_{z}\left(\mu_{s} B\right)^{2}}{2\left(d_{z}+\tilde{J}\right)}} \frac{1}{K\left(\hat{b}_{1} / \hat{b}_{2}\right)},
$$

with $\left(\hat{b}_{1} / \hat{b}_{2}\right)^{2}=\left(J\left(\mu_{s} B\right)^{2}\right) /\left(4 d_{z}\left(d_{z}+\tilde{J}\right)^{2}-2 d_{z}\left(\mu_{s} B\right)^{2}\right)$.

The inertial switching can now be fully described. For an initial excitation with $B>B_{\text {min }}$ [Fig. 2(a)] and a duration time $\Delta t \approx T^{B} / 2=\pi / \omega^{B}$ the system accumulates enough energy $\left(E>2 d_{z}\right)$ to start a precessive switching, as shown in Fig. 2(b) with frequency $\omega_{2}^{\mathrm{AFMR}}$. Due to damping effects, the energy in the system decays until $E<2 d_{z}$, and the precession turns into a precession around the easy axis [Fig. 2(c)] with frequency $\omega_{1}^{\text {AFMR }}$. Depending on the state at which the threshold energy $E=2 d_{z}$ is reached, the sublattices relax into a switched state or the initial state.

For a more quantitative analysis, we simulate the antiferromagnet, $\mathrm{NiO}$, used in the recent experiments with $\mathrm{THz}$ field pulses [11]. We model the $\mathrm{NaCl}$-type crystal structure of $\mathrm{NiO}$ where the magnetic $\mathrm{Ni}^{2+}$ ions are arranged on a cubic fcc lattice and the nonmagnetic $\mathrm{O}^{2-}$ ions are placed in between. Below the Néel temperature $T_{\mathrm{N}}=523 \mathrm{~K}$, the spins of the $\mathrm{Ni}^{2+}$ ions are coupled ferromagnetically in the $\{111\}$ planes and point along the $\langle 11 \overline{2}\rangle$ axis. Neighboring $\{111\}$ planes are ordered antiferromagnetically. We use a similar Hamiltonian as given in Eq. (1) but with two uniaxial hard axes with anisotropy constant $d_{x}=$ $-48.7 \mu \mathrm{eV}$ and $d_{y}=-2.6 \mu \mathrm{eV}$, where $x$ denotes the out of plane direction, $\langle 111\rangle$, and $y$ the in-plane hard axis, $\langle\overline{1} 10\rangle$. The exchange constant for $\mathrm{NN}$ is $J^{\mathrm{NN}}=$ $-1.37 \mathrm{meV}$ and for NNN $J^{\mathrm{NNN}}=19 \mathrm{meV}$. For the magnetic moment, we use $\mu_{s}=2.04 \times 10^{-23} \mathrm{~J} / \mathrm{T}$. These material parameters are known from neutron scattering measurements [14]. 


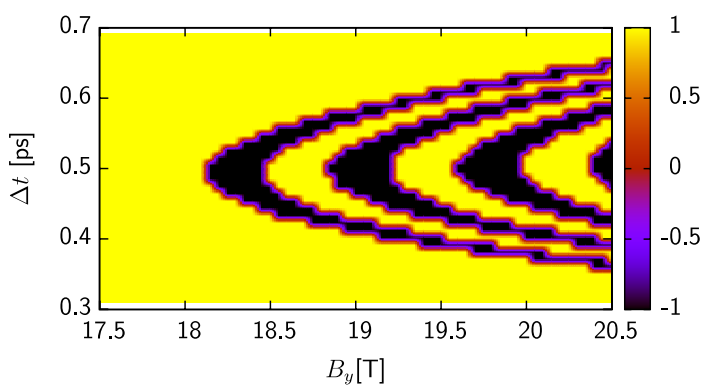

FIG. 3 (color online). $\quad l_{z}$ after excitation of $\mathrm{NiO}$ with an ultrashort magnetic field pulse of different duration and strength.

Figure 3 shows the value of $l_{z}$ after having excited $\mathrm{NiO}$ with a subpicosecond, rectangular magnetic field pulse of different strengths and durations. In the yellow areas the system relaxed back into its initial equilibrium state, $l_{z}=1$, while in the black areas the value of $l_{z}$ after relaxation is negative, indicating switching of the sublattices. In the first black area, around $B_{y}=18.3 \mathrm{~T}$ and $\Delta t=0.49 \mathrm{ps}$, the excitation was sufficient to switch the sublattice magnetization once, as shown earlier in Fig. 1. The field value is a little higher than the minimum field calculated with Eq. (12), which, for an effective exchange constant $\tilde{J}=$ $6\left(J^{\mathrm{NNN}}+J^{\mathrm{NN}}\right)$, would lead to a value of $B_{\min }=17.9 \mathrm{~T}$. The optimal pulse duration for a field pulse around $18 \mathrm{~T}$ calculated with Eq. (13) is $\Delta t_{\mathrm{opt}}=T^{B} / 2=0.49 \mathrm{ps}$, which agrees perfectly with the simulations. For higher excitation, in the second yellow area the damping of the system is not sufficient to catch the system in the new energy minimum and the system relaxes back into its initial state. In the second black area, around $19.1 \mathrm{~T}$ and $0.5 \mathrm{ps}$, there is enough energy in the system for more than one full precession as shown in Fig. 2(b), leading finally to switching. Overall, one can identify a hierarchy of black areas indicating switching after an increasing number of precessions.

Antiferromagnets show no net magnetization, which makes them less important for applications in data storage or spintronics. Since the existence of at least two sublattices with a strong antiferromagnetic coupling is central for the fast dynamics and the inertial switching described above, ferrimagnets could be considered as important an alternative. They also have two sublattices with a strong, antiferromagnetic coupling, but in addition, a measurable total magnetization. Therefore, we investigate the possibility of inertial switching in ferrimagnets as well. Our atomistic spin model for a ferrimagnet is very similar to the antiferromagnetic model described by Eq. (1). We simulate a cubic lattice of about $40 \times 40 \times 40$ spins with periodic boundary conditions. We distinguish between sublattices $A$ and $B$, and set the ratio of the magnetic moments to $\mu_{s}^{A}=$ $2 \mu_{s}^{B}$ with an antiferromagnetic NN coupling $\left(J^{\mathrm{NN}}>0\right)$, ferromagnetic NNN coupling $\left(J_{A}^{\mathrm{NNN}}=3 J_{B}^{\mathrm{NNN}}=-3 J^{\mathrm{NN}}\right)$, anisotropy constant $d_{z}=0.005 J^{\mathrm{NN}}$, and $\alpha=0.05$. These parameters result in a magnetization compensation point

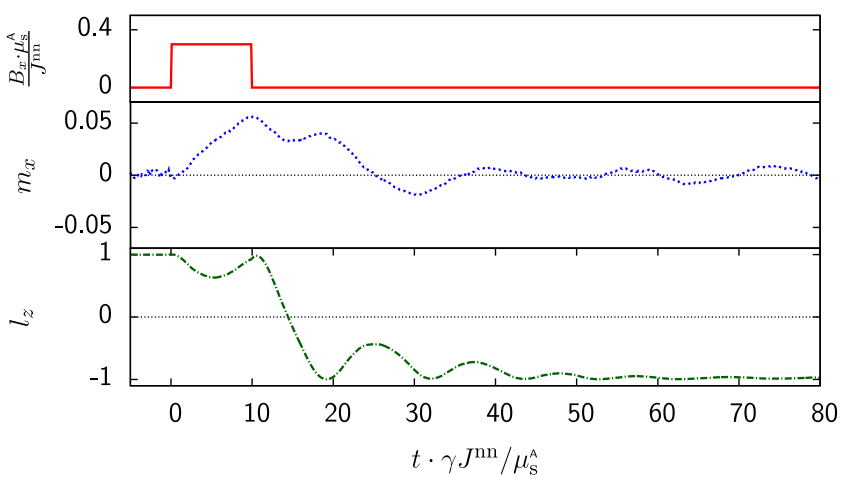

FIG. 4 (color online). Switching of a ferrimagnet at $T_{M}$ with an ultrashort magnetic field pulse.

$T_{\mathrm{M}}=0.7 T_{\mathrm{C}}$, where $T_{\mathrm{C}}$ is the Curie temperature. Our simulations show that the dynamics of a ferrimagnet in the ground state are much more complicated than the dynamics of an antiferromagnet due to the existence of ferromagnetic excitation modes. Nevertheless, Fig. 4 shows a simulation near $T_{\mathrm{M}}$, where we could observe an inertial switching similar to the antiferromagnetic switching we discussed before.

To summarize, we showed that antiferromagnets can be switched all-optically on a time scale of picoseconds using $\mathrm{THz}$ laser radiation. This switching mechanism rests on the coordinated dynamics of the two interacting sublattices and has an inertial character. We calculate the AFMR frequencies in the nonlinear regime, the orbits, and estimate the field strength and optimal pulse duration times required for switching analytically. By means of computer simulations, we demonstrated that ferrimagnets can be switched similarly at their compensation point. The field values needed for switching turn out to be rather large. However, one should note that real $B$ fields from laser radiation are neither rectangular nor single cycle. Hitting the resonance frequency of the antiferromagnet or ferrimagnet [11], the ac magnetic field leads to an increase of the exchange energy with every period. Initial simulations show that more realistic pulse shapes similar to the pulses used in Ref. [11] can already lead to a reduction of the needed field strength for switching by $20 \%-30 \%$. We observed a drastic reduction of the field strength needed for switching in case of longer resonant excitations when the frequency is corrected for its amplitude dependence in the nonlinear regime, as shown in Eq. (10).

Funding by the Center for Applied Photonics Konstanz is gratefully acknowledged.

[1] E. Beaurepaire, J.-C. Merle, A. Daunois, and J. Y. Bigot, Phys. Rev. Lett. 76, 4250 (1996).

[2] C. D. Stanciu et al., Phys. Rev. Lett. 99, 047601 (2007).

[3] K. Vahaplar et al., Phys. Rev. Lett. 103, 117201 (2009). 
[4] N. Kazantseva, D. Hinzke, R. W. Chantrell, and U. Nowak, Europhys. Lett. 86, 27006 (2009).

[5] A. Kirilyuk, A. V. Kimel, and T. Rasing, Rev. Mod. Phys. 82, 2731 (2010).

[6] C. Kittel, Phys. Rev. 82, 565 (1951).

[7] F. Keffer and C. Kittel, Phys. Rev. 85, 329 (1952).

[8] A. V. Kimel et al., Nat. Phys. 5, 727 (2009).

[9] F. Blanchard et al., Opt. Express 15, 13212 (2007).

[10] K.-L. Yeh, M. C. Hoffmann, J. Hebling, and K. A. Nelson, Appl. Phys. Lett. 90, 171121 (2007).
[11] T. Kampfrath et al., Nat. Photonics 5, 31 (2011).

[12] U. Nowak, in Handbook of Magnetism and Advanced Magnetic Materials, edited by $\mathrm{H}$. Kronmüller and $\mathrm{S}$. Parkin (John Wiley \& Sons Ltd., Chichester, 2007), Vol. 2.

[13] See Supplemental Material at http://link.aps.org/ supplemental/10.1103/PhysRevLett.108.247207 for more details of the analytical derivations and a movie of the switching process.

[14] M. Hutchings and E. Samuelsen, Phys. Rev. B 6, 3447 (1972). 